\title{
Probing charm hadronisation with ALICE at the LHC
}

\author{
Cristina Bedda*† \\ Utrecht University \\ E-mail: cristina.beddalcern.ch
}

Charm-quark hadronisation can be investigated by measuring the relative abundance of various particle species, in particular non-strange $\mathrm{D}$ mesons $\left(\mathrm{D}^{0}, \mathrm{D}^{+}, \mathrm{D}^{*+}\right), \mathrm{D}_{\mathrm{s}}^{+}$mesons, and charm baryons $\left(\Lambda_{\mathrm{c}}^{+}, \Xi_{\mathrm{c}}^{0}\right)$. The high precision tracking, excellent vertexing capabilities and particle identification granted by the ALICE apparatus allows hadrons containing charm quarks to be measured over a wide momentum range in $\mathrm{pp}$ and $\mathrm{pA}$ collisions. Measurements of the charmed-baryon production in small systems are also a fundamental reference for heavy-ion collisions, where an enhancement of the baryon-to-meson ratio could derive from hadronisation via coalescence of charm quarks with the quarks in the Quark-Gluon Plasma.

In this contribution, recent measurements of charmed meson and baryon production in pp collisions and in $\mathrm{p}-\mathrm{Pb}$ collisions with the ALICE experiment are presented and compared with theoretical calculations. The results include the $p_{\mathrm{T}}$-differential cross section of $\Lambda_{\mathrm{c}}^{+}$and $\Xi_{\mathrm{c}}^{0}$ baryons, and the baryon-meson ratio $\Lambda_{\mathrm{c}}^{+} / \mathrm{D}^{0}$. The measured values of $\Lambda_{\mathrm{c}}^{+} / \mathrm{D}^{0}$ baryon-meson ratio are significantly higher than expected from model calculations and previous measurements at $\mathrm{e}^{+} \mathrm{e}^{-}$and ep colliders.

The 39th International Conference on High Energy Physics (ICHEP2018)

4-11 July, 2018

Seoul, Korea

\footnotetext{
* Speaker.

${ }^{\dagger}$ On behalf of the ALICE Collaboration.
} 


\section{Introduction}

Heavy-flavour production measurements in pp collisions can be used to test perturbative QCD calculations and to study the fragmentation processes by measuring the relative abundance of various particle species. In $\mathrm{p}-\mathrm{Pb}$ collisions, instead, they can be used to study cold-nuclear-matter effects that can affect the charm/beauty hadron production. In addition, they provide a reference to study the hadronisation processes in $\mathrm{Pb}-\mathrm{Pb}$ collisions.

Charmed hadrons are reconstructed with the ALICE detector [1] at mid-rapidity via hadronic $\left(\mathrm{D}^{0} \rightarrow \mathrm{K}^{-} \pi^{+}, \mathrm{D}^{+} \rightarrow \mathrm{K}^{-} \pi^{+} \pi^{+}, \mathrm{D}^{*+} \rightarrow \mathrm{D}^{0} \pi^{+}, \mathrm{D}_{\mathrm{s}}^{+} \rightarrow \phi \pi^{+} \rightarrow \mathrm{K}^{+} \mathrm{K}^{-} \pi^{+}, \Lambda_{\mathrm{c}}^{+} \rightarrow \mathrm{pK}^{-} \pi, \Lambda_{\mathrm{c}}^{+} \rightarrow \mathrm{pK}_{\mathrm{s}}^{0}\right)$ and semi-leptonic decay channels $\left(\Lambda_{\mathrm{c}}^{+} \rightarrow \mathrm{e}^{+} \Lambda \nu_{\mathrm{e}}\right.$ and $\left.\Xi_{\mathrm{c}}^{0} \rightarrow \mathrm{e}^{+} \Xi^{-} \nu_{\mathrm{e}}\right)$. Details on the reconstruction procedure, the signal extraction and the cross-section evaluation can be found in $[2,3,4]$.

\section{Charmed meson production}
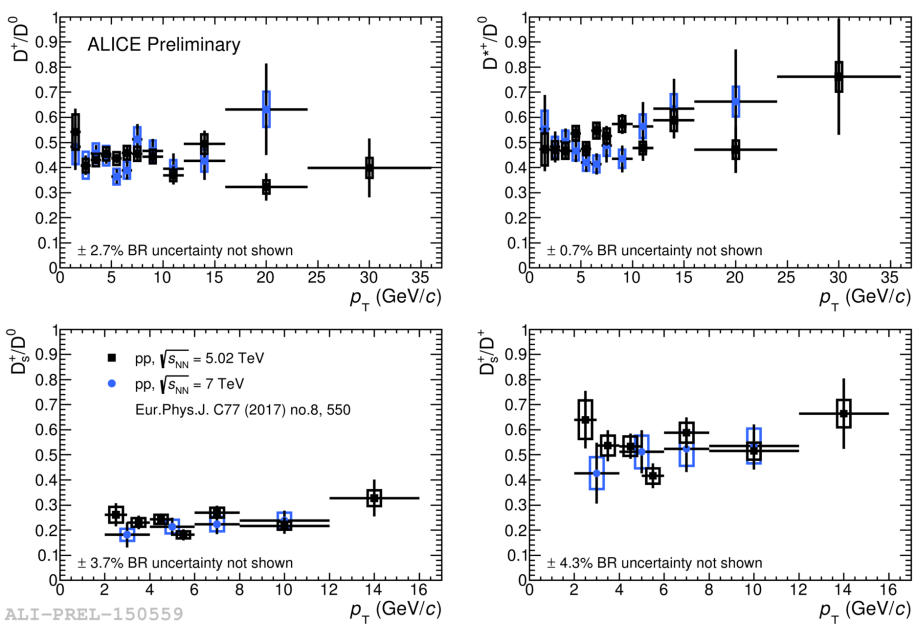

Figure 1: Ratio of prompt D-meson cross-sections in pp collisions at $\sqrt{s}=5.02 \mathrm{TeV}$ and $\sqrt{s}=7 \mathrm{TeV}$ [2]

The ratio of the $p_{\mathrm{T}}$-differential cross sections of $\mathrm{D}^{0}, \mathrm{D}^{+}, \mathrm{D}^{*+}$ and $\mathrm{D}_{\mathrm{s}}^{+}$in pp collisions at $\sqrt{s}=5.02 \mathrm{TeV}$ is shown in Fig. 1. The data sample consists of 990 million of minimum bias events collected in 2017. The measured D-meson ratios do not show a significant $p_{\mathrm{T}}$ dependence within the experimental uncertainties, thus suggesting a small difference between the fragmentation functions of charm quarks to pseudoscalar $\left(\mathrm{D}^{0}, \mathrm{D}^{+}\right.$, and $\left.\mathrm{D}_{\mathrm{s}}^{+}\right)$and vector $\left(\mathrm{D}^{*+}\right)$ mesons and to strange and non-strange mesons. The results are compatible within uncertainties with the corresponding D-meson ratios measured in pp collisions at $\sqrt{s}=7 \mathrm{TeV}$ [2].

The modification of the $\mathrm{D}$-meson yield in $\mathrm{p}-\mathrm{Pb}$ collisions with respect to pp collisions is quantified by the nuclear modification factor $R_{\mathrm{pPb}}=\frac{1}{A} \frac{\mathrm{d}^{2} \sigma_{\mathrm{pPb}}^{\text {promptD }} / \mathrm{d} p_{\mathrm{T}} \mathrm{d} y}{\mathrm{~d}^{2} \sigma_{\mathrm{pp}}^{\text {promptD }} / \mathrm{d} p_{\mathrm{T}} \mathrm{d} y}$, where $\mathrm{A}=208$ is the $\mathrm{Pb}$ mass number. The pp cross section is obtained from the data collected in 2017. The $\mathrm{p}-\mathrm{Pb}$ data sample consists of 600 million minimum bias events collected in 2016. The $R_{\mathrm{pPb}}$ of non-strange D mesons, resulting from the average of $\mathrm{D}^{0}, \mathrm{D}^{+}$and $\mathrm{D}^{*+}$, is reported in Fig. 2 (left). The data are compatible with unity within uncertainties and can be described in the intermediate $p_{\mathrm{T}}$ region by models including cold-nuclear-matter effects and the formation of a small Quark-Gluon Plasma in $\mathrm{p}-\mathrm{Pb}$ collisions. 
The data disfavor a suppression at high $p_{\mathrm{T}}$, however the uncertainties of the data are large and this does not allow a discrimination between models. A more detailed description of the models can be found in [5]. Fig 2 (right) shows the comparison of the strange and non-strange D meson $R_{\mathrm{pPb}}$ that are compatible within the uncertainties.
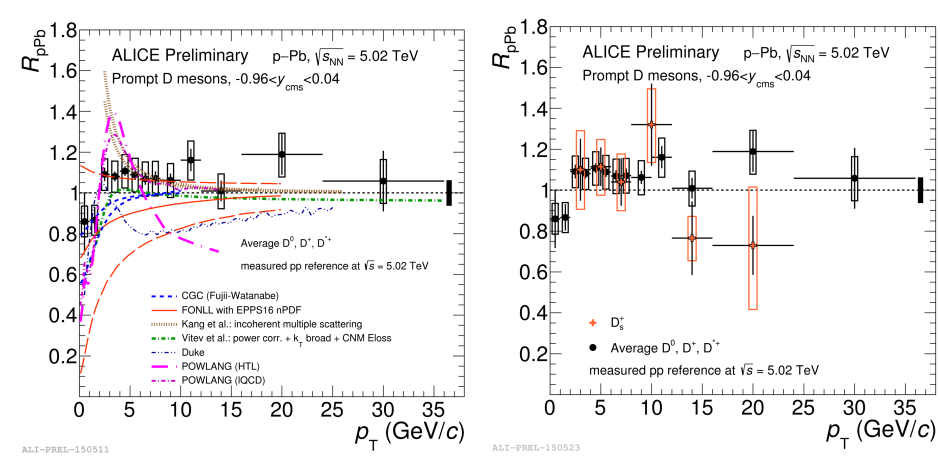

Figure 2: $R_{\mathrm{pPb}}$ of non-strange $\mathrm{D}$ mesons compared with theoretical calculations (left) and the $\mathrm{D}_{\mathrm{s}}^{+}$-meson $R_{\mathrm{pPb}}$ (right) in $\mathrm{p}-\mathrm{Pb}$ collisions at $\sqrt{s_{\mathrm{NN}}}=5.02 \mathrm{TeV}$.

\section{Charmed baryon production and baryon-to-meson ratio}
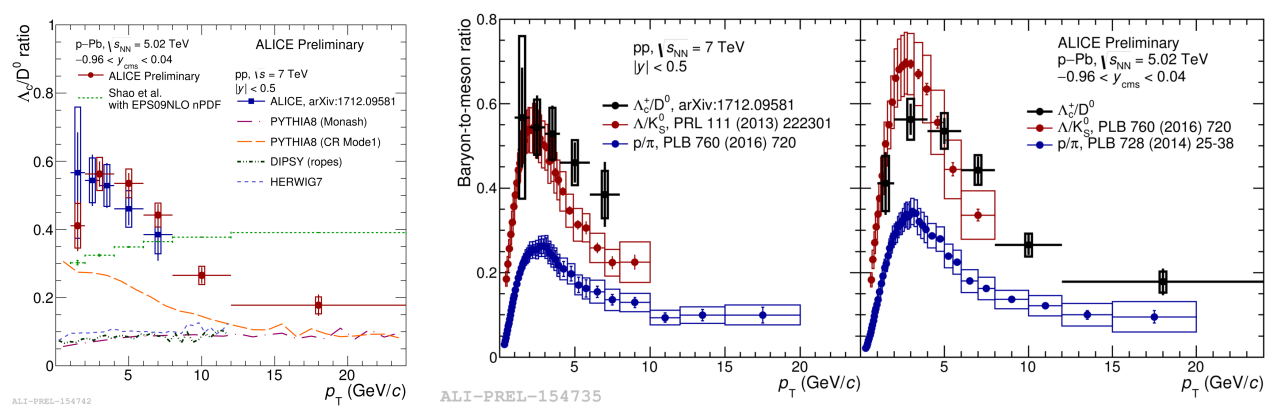

Figure 3: $\Lambda_{\mathrm{c}}^{+} / \mathrm{D}^{0}$ ratio in pp collisions at $\sqrt{s}=7 \mathrm{TeV}$ [4] and in $\mathrm{p}-\mathrm{Pb}$ collisions at $\sqrt{s_{\mathrm{NN}}}=5.02 \mathrm{TeV}$ compared with theoretical calculations $[6,7,9,8]$ and $\Lambda / \mathrm{K}_{\mathrm{s}}^{0}[10]$ and $\mathrm{p} / \pi[11]$ ratios.

The baryon-to-meson ratio $\Lambda_{\mathrm{c}}^{+} / \mathrm{D}^{0}$ is shown in Fig. 3 (left) for pp and $\mathrm{p}-\mathrm{Pb}$ collisions in comparison with models. The results in pp collisions have been published (together with the $\Lambda_{\mathrm{c}}^{+}$ $p_{\mathrm{T}}$-differential cross section) in [4]. The results in $\mathrm{p}-\mathrm{Pb}$ collisions are obtained with the data collected in 2016 that allows an improved precision and extended coverage with respect to the previous measurement [4]. The results are compatible within the relatively large pp uncertainties and the measured $\Lambda_{c}^{+} / D^{0}$ ratio are higher than the expectation from theoretical models: PYTHIA8 with Monash tune and with a tune with enhanced colour reconnection [6], DIPSY with ropes [7], HERWIG7 with a cluster hadronisation mechanism [9], and a calculation using the data-driven model tuned on LHCb pp data at forward rapidity [8]. The later calculations is closer to the data at low $p_{\mathrm{T}}$ but can not reproduce the observed trend. PYTHIA8 with enhanced colour reconnection gets closer to the data, hinting that the role of colour reconnection can be relevant in charm hadronisation. The integrated $\Lambda_{\mathrm{c}}^{+} / \mathrm{D}^{0}$ ratio measured by ALICE is also higher than previous measurements 
in $\mathrm{e}^{+} \mathrm{e}^{-}$and ep collisions at lower centre-of-mass energies. In Fig. 3 (right), the baryon-to-meson ratio in the charm sector is compared with the same ratios in the light flavour sectors $\left(\Lambda / K_{\mathrm{s}}^{0}[10]\right.$ and $\mathrm{p} / \pi[11])$. Within the relatively large uncertainties, a similar $p_{T}$-trend with decreasing values from $p_{\mathrm{T}}=4 \mathrm{GeV} / c$ is observed in the two sectors.

The first measurements of the $\Xi_{\mathrm{c}}^{0}$ cross section at the LHC and $\Xi_{\mathrm{c}}^{0} / \mathrm{D}^{0}$ ratio are also reported in Fig. 4. On the right panel, the $\Xi_{\mathrm{c}}^{0} / \mathrm{D}^{0}$ ratio is compared with PYTHIA8 models, the results are underestimated by the expectations as for the $\Lambda_{\mathrm{c}}^{+} / \mathrm{D}^{0}$ ratio.
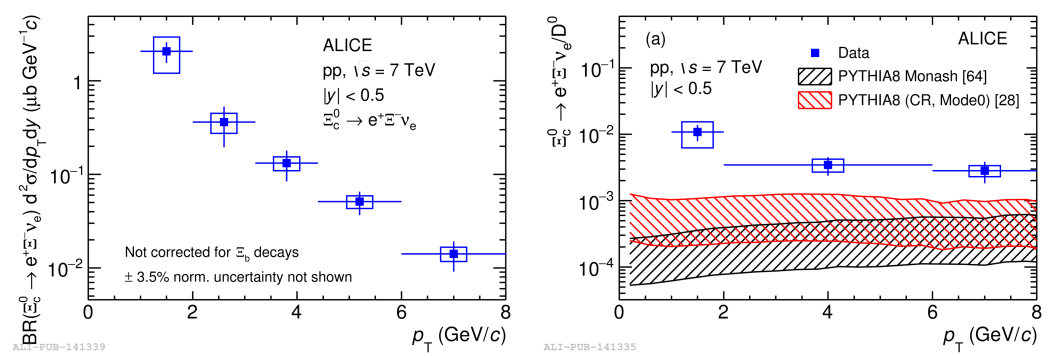

Figure 4: Cross-section of $\Xi_{\mathrm{c}}^{0}$ and $\Xi_{\mathrm{c}}^{0} / \mathrm{D}^{0}$ ratio compared with theoretical calculations in pp collisions at $\sqrt{s}=7 \mathrm{TeV}[3]$.

\section{Summary}

The ALICE results on charm-meson and charm-baryon production have been reported. The Dmeson particle ratios are compatible between $\mathrm{pp}$ and $\mathrm{p}-\mathrm{Pb}$ collisions. The strange and non-strange D meson $R_{\mathrm{pPb}}$ are compatible within uncertainties. The $\Lambda_{\mathrm{c}}^{+} / \mathrm{D}^{0}$ ratio in $\mathrm{pp}$ and $\mathrm{p}-\mathrm{Pb}$ collisions is higher than the theoretical expectations and it has a similar $p_{\mathrm{T}}$-trend as the baryon-to-meson ratios in the light-flavour sector. The first measurement of the $\Xi_{c}^{0}$ cross section at the LHC has also been reported and the $\Xi_{\mathrm{c}}^{0} / \mathrm{D}^{0}$ baryon-to-meson ratio has been found to be higher than model expectations.

\section{References}

[1] ALICE Collaboration, JINST 3(2008)S08002.

[2] ALICE Collaboration, Eur. Phys. J. C77 (2017) no.8, 550.

[3] ALICE Collaboration, Phys.Lett. B 781. (2018) 8.

[4] ALICE Collaboration, JHEP 04 (2018) 108, JHEP 1804 (2018) 108.

[5] ALICE Collaboration, ALICE-PUBLIC-2017-008.

[6] J. R. Christiansen and P. Z. Skands, JHEP 1508(2015)003.

[7] J. R. C. Christian Bierlich, Phys. Rev. D92(2015)no. 92.

[8] HS. Shao et al, Eur. Phys. J. C77, no. 1, 1 (2017)

[9] M. Bahr. et al., Eur. Phys. J. C58 (2008) 639.

[10] ALICE Collaboration,Phys. Rev. Lett. 111 no. 10, (2013) 222301.

[11] ALICE Collaboration,Phys. Lett. B760 (2016) 720. 